\title{
Audiological Evaluation of Affected Members from a Dutch DFNA8/12 (TECTA) Family
}

\author{
Rutger F. Plantinga, Cor W. R. J. Cremers, Patrick L. M. Huygen, Henricus P. M. Kunst, \\ and Arjan J. Bosman \\ Department of Otorhinolaryngology, Radboud University Nijmegen Medical Centre, Nijmegen, \\ The Netherlands
}

Received: 12 August 2006; Accepted: 1 October 2006; Online publication: 30 November 2006

\begin{abstract}
In DFNA8/12, an autosomal dominantly inherited type of nonsyndromic hearing impairment, the TECTA gene mutation causes a defect in the structure of the tectorial membrane in the inner ear. Because DFNA8/12 affects the tectorial membrane, patients with DFNA8/12 may show specific audiometric characteristics. In this study, five selected members of a Dutch DFNA8/12 family with a TECTA sensorineural hearing impairment were evaluated with pure-tone audiometry, loudness scaling, speech perception in quiet and noise, difference limen for frequency, acoustic reflexes, otoacoustic emissions, and gap detection. Four out of five subjects showed an elevation of pure-tone thresholds, acoustic reflex thresholds, and loudness discomfort levels. Loudness growth curves are parallel to those found in normalhearing individuals. Suprathreshold measures such as difference limen for frequency modulated pure tones, gap detection, and particularly speech perception in noise are within the normal range. Distortion otoacoustic emissions are present at the higher stimulus level. These results are similar to those previously obtained from a Dutch DFNA13 family with midfrequency sensorineural hearing impairment. It seems that a defect in the tectorial membrane results primarily in an attenuation of sound, whereas suprathreshold measures, such as otoacoustic emissions and speech perception in noise, are preserved rather well. The main effect of the defects is a shift in the operation point of the outer hair cells
\end{abstract}

Correspondence to: Arjan J. Bosman • Radboud University Nijmegen Medical Centre - Nijmegen, The Netherlands. Telephone: +31-243614947; fax: +31-24-3614926; email: a.bosman@kno.umcn.nl with near intact functioning at high levels. As most test results reflect those found in middle-ear conductive loss in both families, the sensorineural hearing impairment may be characterized as a cochlear conductive hearing impairment.

Keywords: DFNA8/12, TECTA, DFNA13, sensorineural hearing impairment, psychophysics

\section{INTRODUCTION}

Since 1992, more than 100 types of sensorineural, nonsyndromic hearing impairment have been identified (Van Camp and Smith 2006). The different chromosomal loci for nonsyndromic types of hearing impairment have been designated DFN (deafness) and are numbered in chronological order of discovery. Autosomal dominant types are referred to as DFNA, autosomal recessive types as DFNB, and Xlinked types as DFN. These types of hearing impairment can be characterized by age of onset, presence and degree of progression, severity of hearing impairment, and audiometric configuration (Huygen et al. 2006). One of these configurations is the Ushaped or "cookie-bite" audiometric configuration of midfrequency hearing impairment that can be found in DFNA8/12 (TECTA) and DFNA13 (COL11A2) (Van Camp and Smith 2006).

The first descriptions of autosomal dominant midfrequency hearing impairment relate to an Austrian (DFNA8) and a Belgian (DFNA12) family (Kirschhofer et al. 1998; Verhoeven et al. 1997). The original proposed linkage to chromosome 15q in the Austrian family was later on withdrawn and relocated to chromosome 11q22-24 (Kirschhofer 


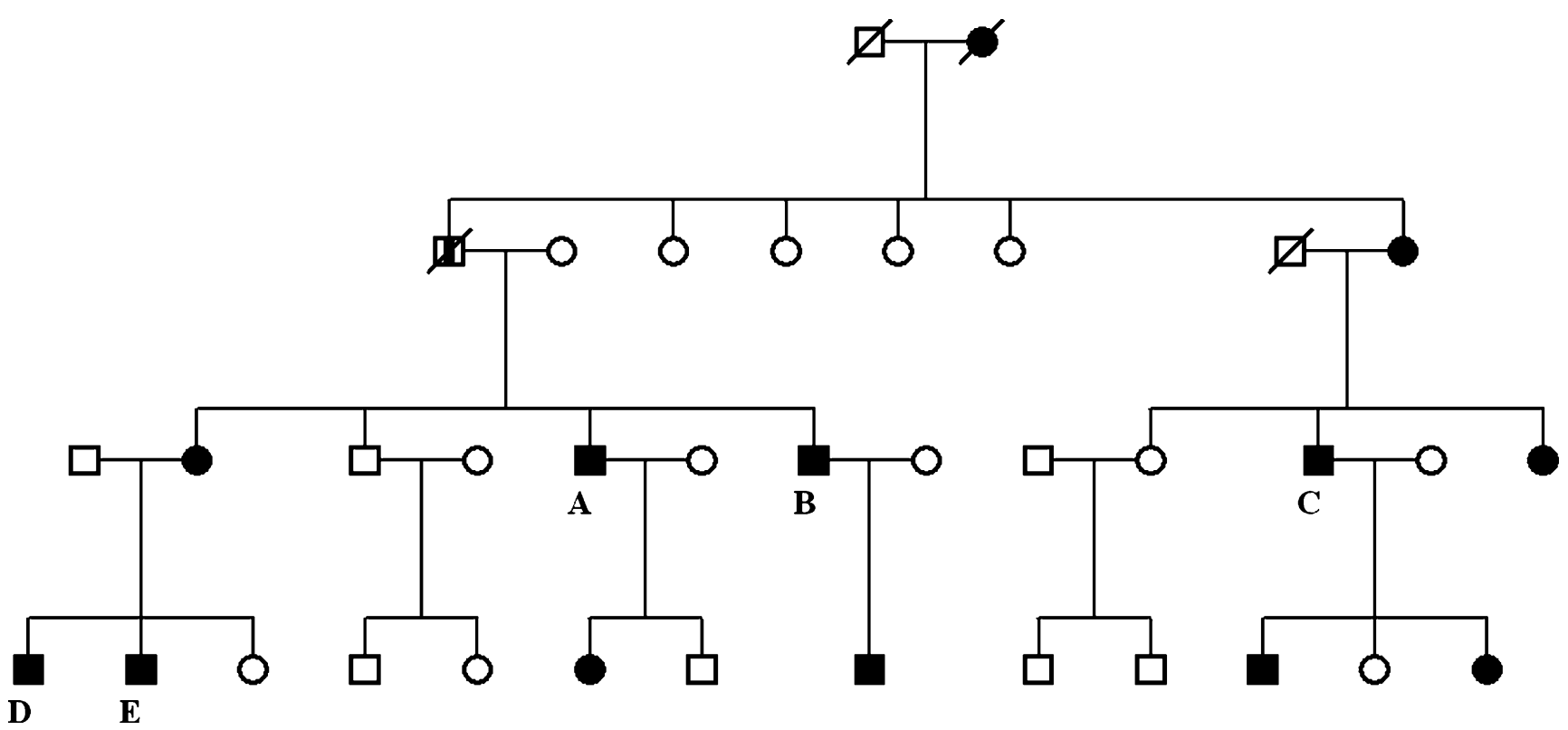

FIG. 1. Pedigree of the Dutch DFNA8/12 (TECTA) family. Subjects participating in this study are indicated as (A), (B), (C), (D), and (E) (Plantinga et al. 2006).

et al. 1998; Verhoeven et al. 1997, 1998). When the mutations proved to be in the same gene (TECTA), which encodes alpha-tectorin, the locus was redesignated DFNA8/12 (Mustapha et al. 1998).

Alpha-Tectorin is an important noncollagenous component of the tectorial membrane in the cochlea and its level of expression is high during tectorial membrane morphogenesis, between the 12th and 20th weeks of embryonic development, after which it decreases dramatically (Pfister et al. 2004). The tectorial membrane consists of an extracellular matrix overlying the organ of Corti that contacts the outer hair cells and plays an important role in intracochlear sound transmission by ensuring optimal cochlear feedback (Legan et al. 2000). Research on transgenic mice showed that the tectorial membrane facilitates the motion of the basilar membrane to optimally drive the inner hair cells (Legan et al. 2005). The alpha-tectorin protein comprises three distinct modules: the entactin G1 domain, the zonadhesin (ZA) domain, and the zona pellucida (ZP) domain (Legan et al. 1997). Mutations affecting the $\mathrm{ZP}$ domain are significantly associated with midfrequency hearing impairment, whereas mutations in the ZA domain are significantly associated with high frequency hearing impairment (Plantinga et al. 2006). Furthermore, mutations in either domain causing substitution of cysteine residues are significantly associated with progressive hearing impairment (Plantinga et al. 2006).

Legan et al. (2005) studied mice with a mutation in the ZP domain of TECTA. In these mice with a heterozygous Y1870C mutation $\left(\right.$ Tecta $\left.{ }^{\mathrm{Y} 1870 \mathrm{C} /+}\right)$, the tectorial membrane's matrix structure is disrupted and its adhesion zone is reduced in thickness (Legan et al. 2005). These changes do not seriously influence the tectorial membrane's role in ensuring optimal cochlear feedback; however, neural tuning is broadened and a decrease in sensitivity is observed at the tip of the neural tuning curve (Legan et al. 2005).

In 2003, De Leenheer et al. studied the audiological characteristics of affected members of a Dutch DFNA13 family (De Leenheer et al. 2004). DFNA13 is another type of autosomal dominant midfrequency hearing impairment, which is caused by a mutation of COL11A2 on chromosome $6 p$ that also results in alterations of the tectorial membrane (McGuirt et al. 1999). In this DFNA13 family, hearing impairment was nonprogressive (De Leenheer et al. 2004). They observed that in younger subjects suprathreshold signal analyzing capacities are not compromised by the moderate sensorineural hearing impairment, and concluded that DFNA13 results in a cochlear type of conductive hearing impairment (De Leenheer et al. 2004).

In the present DFNA8/12 (TECTA) family, nonprogressive midfrequency hearing impairment is caused by a missense mutation substituting arginine residues (R1890C) in the $\mathrm{ZP}$ domain of alphatectorin (Plantinga et al. 2006). The presumably congenital hearing impairment predominantly affects the mid frequencies ( 1 and $2 \mathrm{kHz}$ ), with thresholds around $40 \mathrm{~dB}$. Speech recognition was almost perfect with phoneme recognition scores higher than 90\% (Plantinga et al. 2006).

Such patients with a proven and specific defect in the tectorial membrane provide a unique opportunity 

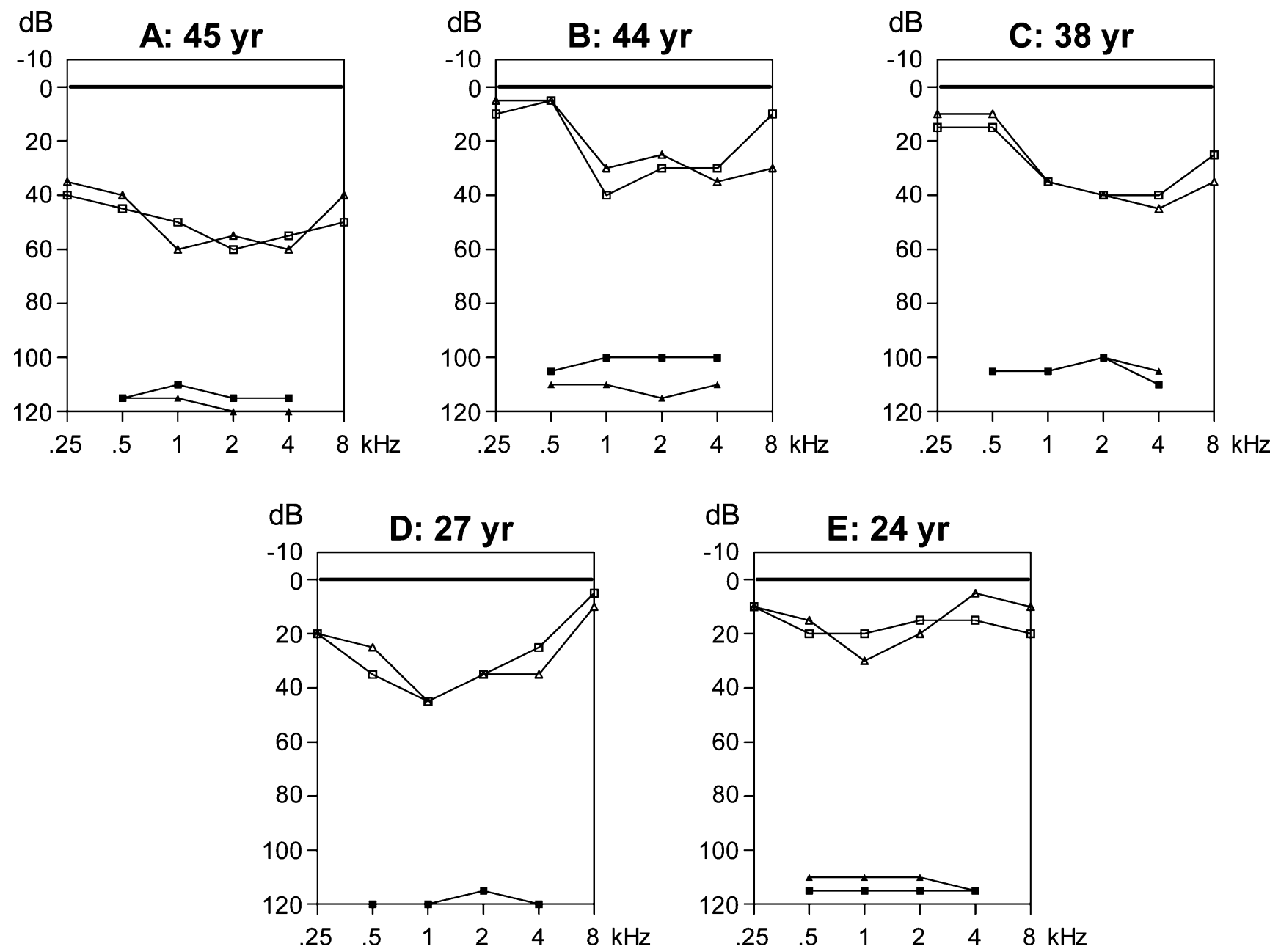

FIG. 2. Individual hearing thresholds and loudness discomfort levels for subjects (A-E). Air conduction thresholds are indicated with open squares (right ear) and triangles (left ear), and loudness discomfort levels are indicated with black squares (right ear) and triangles (left ear).

to study the audiometric characteristics of tectorial membrane defects. To further outline the audiometric phenotype of DFNA8/12, an extended set of audiological tests was performed on selected affected members of a Dutch DFNA8/12 (TECTA) family. As the tectorial membrane is essential both in cochlear transduction and amplification tests were selected that focus on suprathreshold characteristics in the time and frequency domain. Outer hair cell functionality was tested separately with distortion product otoacoustic emissions (DPOAE). Results of these tests will be compared to those previously obtained from members of a Dutch DFNA13 (COL11A2) family (De Leenheer et al. 2004).

\section{METHODS AND MATERIALS}

Five adult members of the Dutch DFNA8/12 (TECTA) family, all with a confirmed TECTA gene mutation, were contacted and agreed to participate in this study (Plantinga et al. 2006). The gene expression was not significantly different for male and female family members. Subjects participating in this study are indicated as A, B, C, D, and E in the pedigree shown in Figure 1. Subjects A to D showed hearing impairment typical for the disorder; subject E only has a relatively mild degree of hearing impairment, but was nevertheless included. Measurements were performed on the ear with the greatest overall impairment.

At the beginning of the experiment, pure-tone thresholds were measured with standard audiometric procedures and equipment (Interacoustics AC-40 audiometer). In addition, loudness discomfort levels were measured to provide safe upper limits of stimulus levels when measuring acoustic reflexes.

Speech perception was measured in quiet with standard monosyllabic Dutch word lists (Bosman and Smoorenburg 1995). Each list consists of 11 CVC syllables and scores are based on correct repetition of phonemes. 


\section{TABLE 1}

Acoustic reflex thresholds in $\mathrm{dB} \mathrm{HL}$ for ipsilateral stimulation (worst ear) with pure-tone stimuli of $0.5,1,2$, and $4 \mathrm{kHz}$

\begin{tabular}{ccccr}
\hline Subject & $0.5 \mathrm{kHz}$ & $1 \mathrm{kHz}$ & $2 \mathrm{kHz}$ & $4 \mathrm{kHz}$ \\
\hline A & 100 & 100 & 105 & $>100$ \\
B & 80 & 90 & 90 & 100 \\
C & 85 & 95 & 95 & 100 \\
D & 85 & 95 & 95 & 95 \\
E & 90 & 95 & 95 & 100 \\
\hline
\end{tabular}

Ipsilateral acoustic reflexes were measured at the pressure providing maximum compliance in the tympanogram (Madsen Zodiac 901 tympanometer).

DPOAE were measured with ILO 292 equipment. The stimuli had a frequency ratio of 1.2, and stimulus levels of 75/60 and 80/70 dB SPL were used. At these levels, artifacts due to nonlinearities in the measurement setup fell below the noise floor. Loudness scaling was performed with a 7-point categorical scale at 500 and $2000 \mathrm{~Hz}$ (Moser 1987). Gap detection was measured with gated white noise and with $500-\mathrm{Hz}$ and $2-\mathrm{kHz}$ noise. Digital filtering of the temporal pattern created the frequency-specific stimuli.

Frequency discrimination was measured with frequency-modulated pure tones generated by an audiometer (Interacoustics AC-40). The modulation frequency ranged between $0.1 \%$ and $5 \%$. Subjects indicated whether their pitch percept was stable (not modulated) or unstable (modulated). At least three trials aiming at $50 \%$ correct performance with a sqrt(2) step size were used. Stimuli were presented at the individual listener's most comfortable level, i.e., about $40 \mathrm{~dB}$ SL.

Speech perception in noise was measured with short, everyday Dutch sentences (Plomp and Mimpen 1979). Speech reception threshold (SRT) was measured with the simple up-down procedure proposed by Plomp and Mimpen (1979). SRT will be expressed as a signal-to-noise ratio. All tests were performed in a double-walled sound-treated room. Data will be

\section{TABLE 2}

Distortion product emission (DPOAE) data with 75/60 and 80/70 dB SPL stimuli, measured with ILO-92 equipment (worst ear)

\begin{tabular}{ccc}
\hline Subject & DPOAE $(75 / 60)(\mathrm{kHz})$ & DPOAE $(80 / 70)(\mathrm{kHz})$ \\
\hline A & n.a. & n.a. \\
B & 4.0 & $2.0-6.0$ \\
C & $1.0,2.8,4.0$ & $1.0-6.0$ \\
D & $1.4,4.0$ & $1.0-6.0$ \\
E & n.a. & $1.0-6.0$ \\
\hline
\end{tabular}

Emissions were tested at 1.0, 1.4, 2.0, 2.8, 4.0, and $6.0 \mathrm{kHz}$. The frequency range at which emissions occur is indicated for each subject.
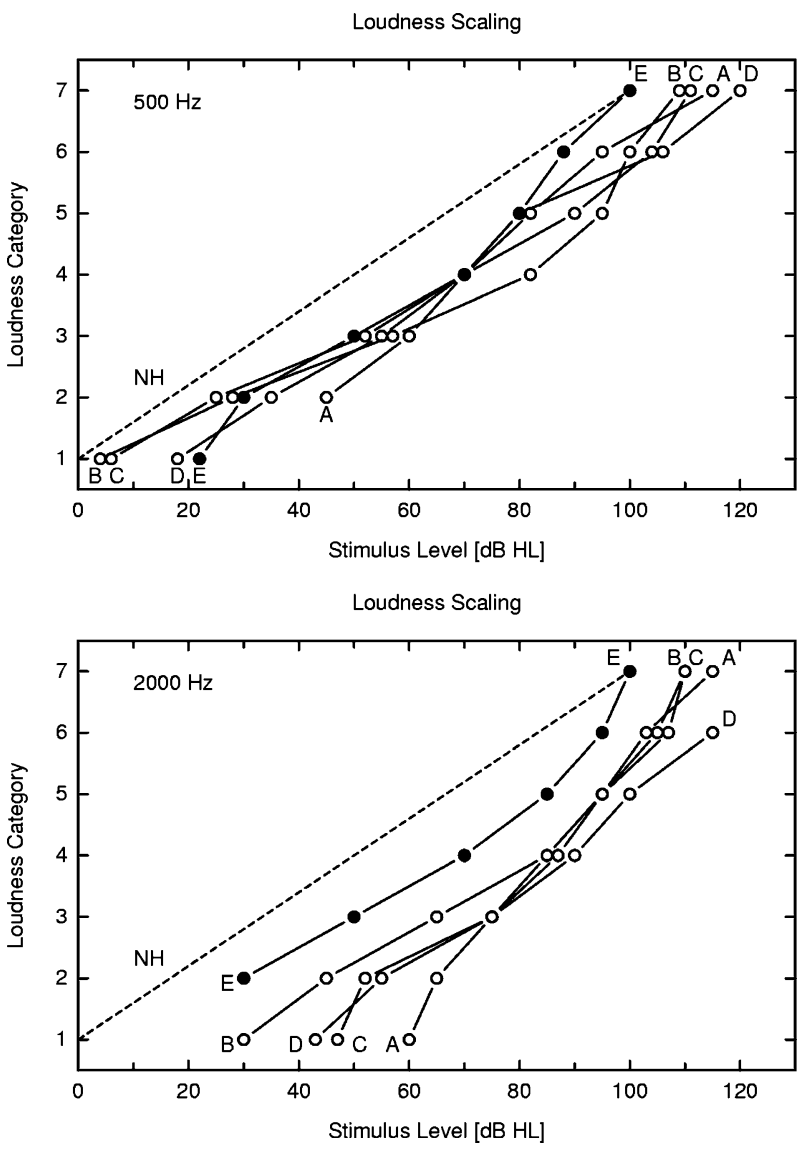

FIG. 3. Loudness growth functions obtained from the worst ear with 7-point categorical scaling (modified Würzburger Hörfeld Skalierung) for $500-\mathrm{Hz}$ and $2-\mathrm{kHz}$ stimuli. Open circles represent scores for subjects (A-D). Scores for subject (E) are represented by black dots.

compared to those for young normal-hearing $(\mathrm{NH})$ listeners.

\section{RESULTS}

Figure 2 shows pure-tone thresholds and loudness discomfort levels on an individual basis. No substantial airborne gaps were observed. Subjects are ordered according to their age. All subjects exhibited a hearing impairment between 1 and $4 \mathrm{kHz}$ ranging from about 30 to $60 \mathrm{~dB}$. In all subjects loudness discomfort levels appeared higher than normal (Pascoe 1988).

Acoustic reflex thresholds obtained with ipsilateral stimulation are shown in Table 1. Most reflex thresholds are elevated compared to the value of $85 \mathrm{~dB} \mathrm{HL}$ for normal hearing (Silman and Gelfand 1981).

The occurrence of DPOAE is shown in Table 2. In subject A, no emissions were measured. At stimulus levels of $80 / 70 \mathrm{~dB}$ SPL emissions were found in the other four subjects; at the lower stimulus level of 75/ $60 \mathrm{~dB}$ SPL, some emissions occurred in subjects B-D. 


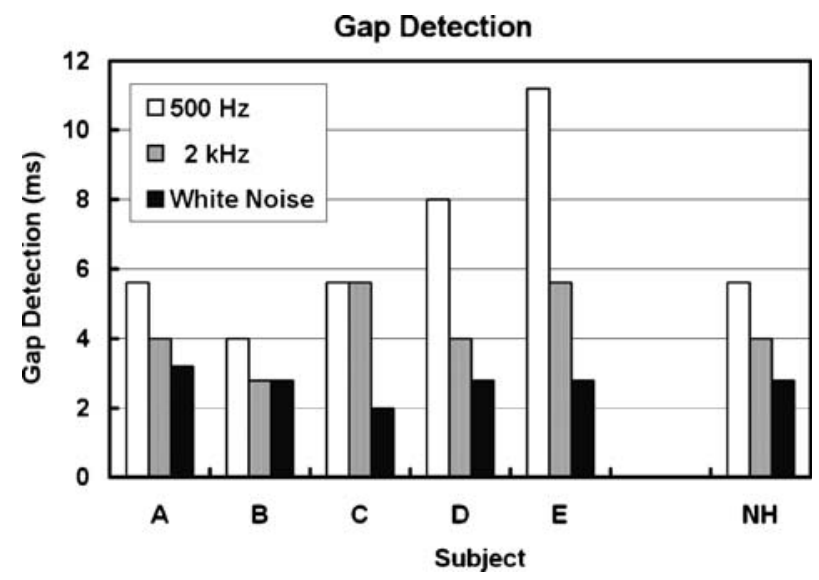

FIG. 4. Gap detection with white noise and with $500-\mathrm{Hz}$ and 2$\mathrm{kHz}$ octave bands of noise. Data are shown for subjects (A-E) (worst ear) and for normal-hearing listeners $(\mathrm{NH})$.

Interestingly, in subject $\mathrm{E}$, who has the most favorable pure-tone thresholds, no emissions occurred at the lowest stimulus level.

Figure 3 shows loudness growth functions for 500$\mathrm{Hz}$ and $2-\mathrm{kHz}$ pure-tone stimuli, obtained with a 7 point categorical scale (Moser 1987). With the exception of the data for subject $\mathrm{E}$, loudness growth curves are more or less parallel to those for $\mathrm{NH}$ subjects, with elevated loudness discomfort levels between 100 and $120 \mathrm{~dB}$ HL. Loudness judgments of subject $\mathrm{E}$ are within normal limits.

Gap detection was measured with $500-\mathrm{Hz}$ and 2$\mathrm{kHz}$ octave bands of white noise, and with unfiltered white noise. Results are shown in Figure 4, together with average data for seven naive NH listeners. The measurement for subjects A-D are close to those of NH subjects, with a possible exception for subject D only at $500 \mathrm{~Hz}$. Subject E showed poor results at 500 $\mathrm{Hz}$ in this test.

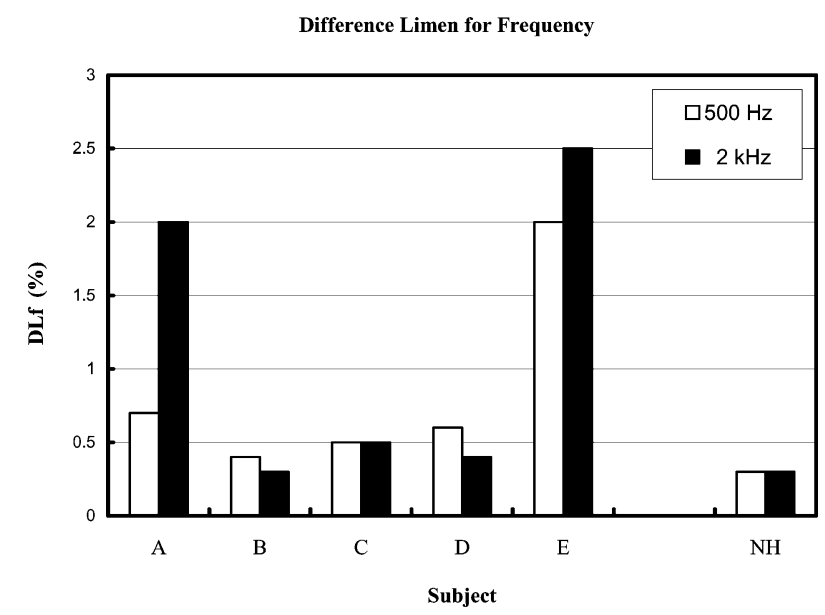

FIG. 5. Difference limen for frequency measured with FMmodulated $500-\mathrm{Hz}$ and $2-\mathrm{kHz}$ stimuli. Data are shown for subjects (A-E) (worst ear) and for normal-hearing $(\mathrm{NH})$ listeners.

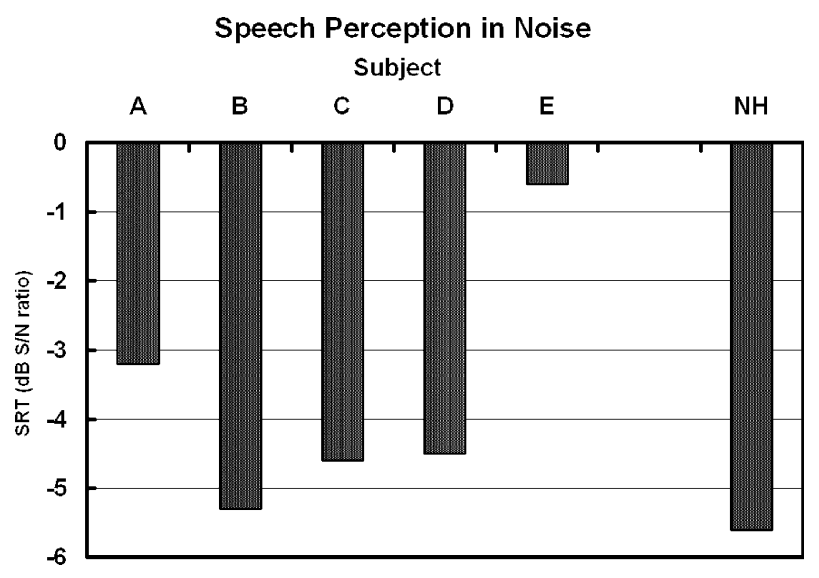

FIG. 6. Speech Reception Thresholds in noise for sentences. Data are shown for subjects (A-E) (worst ear) and for normal-hearing $(\mathrm{NH})$ listeners.

Frequency discrimination as expressed in the difference limen for frequency $\left(\mathrm{DL}_{\mathrm{f}}\right)$ was measured at $500 \mathrm{~Hz}$ and $2 \mathrm{kHz}$. Data are shown in Figure 5, together with data for normal hearing. The data of subjects A-D, except for the $2-\mathrm{kHz}$ data in subject A, are close to those of the normal subjects. Again, subject E performed relatively poorly on this test.

All subjects, with the exception of subject A, had a maximum score of $100 \%$ for speech perception in quiet (data not shown). Figure 6 shows SRT in noise for sentences. The SRTs are expressed as a signal-tonoise ratio. The data for subjects $\mathrm{A}-\mathrm{D}$ are within the range of $\mathrm{NH}$ subjects (normal limit: $-3 \mathrm{~dB}$ ) (Plomp and Mimpen 1979). Alternatively, when expressing the data in Plomp's D (distortion) term values of $+2.4,+0.3,+0.9$, and $+1.1 \mathrm{~dB}$ are found for subjects $\mathrm{A}$, $\mathrm{B}, \mathrm{C}$, and D, respectively, and $+5.1 \mathrm{~dB}$ for subject $\mathrm{E}$. Once more, the data for subject $\mathrm{E}$ are outside the normal range.

\section{DISCUSSION}

Subjects A to D show an elevation of pure-tone thresholds, acoustic reflex thresholds, and loudness discomfort levels. Loudness growth curves are parallel to those found in normal hearing. Suprathreshold measures such as $\mathrm{DL}_{\mathrm{f}}$, gap detection, and particularly speech perception in noise are within the normal range. Distortion otoacoustic emissions are present at the higher stimulus level. These findings suggest normal cochlear function at higher stimulus levels. This contrasts strongly to results normally found in sensorineural hearing impairment resulting from loss of outer and/or inner hair cells (e.g., presbyacusis, noise induced hearing loss) (Moore 1995).

We have no explanation for the relatively poor results found in subject $E$, whereas his pure-tone 
thresholds were the most favorable (Fig. 2). The hearing characteristics of subject $\mathrm{E}$ resemble more closely sensorineural impairment due to hair cell loss. Subject E is thus clearly breaking the trend set by subjects $\mathrm{A}$ to $\mathrm{D}$. In view of the unexpected degree of variation in findings, one might wish to extend the number of reliable observations. Unfortunately, this is not an option for the present family. However, the affected family members that were as yet too young to include in this study may be examined later. Alternatively, additional families with a similar phenotype may be recruited for new studies.

It emerges from the present study that a defect in the tectorial membrane reduces sound transduction, resulting in sound attenuation. Suprathreshold measures such as otoacoustic emissions and speech perception in noise seem to be preserved rather well. The preservation of stimulus fine structure at higher levels is also found in conductive middle ear hearing impairment. This supports the conclusion of De Leenheer et al. (2004) that a conductive cochlear type of hearing impairment occurs in tectorial membrane dysfunction.

The changes observed in the Tecta ${ }^{\mathrm{Y} 1870 \mathrm{C} /+}$ mouse do not seriously influence the tectorial membrane's role in ensuring optimal cochlear feedback; however, neural thresholds were elevated and neural tuning curves were broadened (Legan et al. 2005). Except for the broadening of the neural tuning curves, this Tecta $^{\mathrm{Y1870 \textrm {C } / +}}$ mouse model closely represents the findings in our family. In humans, however, TECTA mostly affects threshold sensitivity with little suprathreshold consequences.

The test results of our subjects are quite similar to the results in subjects with DFNA13 (COL11A2) (De Leenheer et al. 2004). Mice with a targeted disruption of COL11A2 exhibit a loss of organization of the collagen fibrils present in the tectorial membrane, causing pathological alteration in the structure of the tectorial membrane that reduces efficacy of the outer hair cells, resulting in congenital hearing impairment that varies in degree from mild to moderately severe (McGuirt et al. 1999). More data are needed on the suprathreshold hearing characteristics of COL11A2 mice.

In recent years, mouse models provided a lot of insight in the function of the tectorial membrane and the basis of these types of autosomal dominant midfrequency hearing impairment (Legan et al. 1997, 2000, 2005; McGuirt et al. 1999). However, the exact mechanism of mutations altering protein interactions and thus influencing the mechanotransductional properties of the tectorial membrane remains uncertain.

In conclusion, the cochlear loss observed in selected members of a DFNA8/12 family acts purely as an attenuation, a result of a reduced efficiency in the coupling of the outer hair cells and the tectorial membrane. These findings are in line with previous observations in DFNA13 (De Leenheer et al. 2004). Although the underlying mutations have different effects on the structure of the tectorial membrane, the common effect of either disorder may be characterized as a cochlear conductive hearing impairment. More precise consequences of TECTA can only be stated with more certainty after studying affected human temporal bones.

\section{ACKNOWLEDGMENTS}

We wish to thank the participating family members. This project was supported by the European Commission FP6 Integrated Project EUROHEAR, LSHG-CT-20054-512063, and funds from the Heinsius-Houbolt Foundation (to C.W.R.J. Cremers).

\section{REFERENCES}

Bosman AJ, Smoorenburg GF. Intelligibility of Dutch CVC syllables and sentences for listeners with normal hearing and with three types of hearing impairment. Audiology 34:260-284, 1995.

De Leenheer EMr, Bosman AJ, Kunst HPM et Al. Audiological characteristics of some affected members of a Dutch DFNA13/ COL11A2 family. Ann. Otol. Rhinol. Laryngol. 113:922-929, 2004.

Huygen PlM, Pauw RJ, Cremers CWRJ. Audiometric profiles associated with genetic non-syndromic hearing impairment, a review and phenotype analysis. Audiol. Med. 2006.

Kirschhofer K, Kenyon JB, Hoover DM et AL. Autosomal-dominant, prelingual, nonprogressive sensorineural hearing loss: localization of the gene (DFNA8) to chromosome 11q by linkage in an Austrian family. Cytogenet. Cell Genet. 82:126-130, 1998.

Legan PK, Rau A, Keen JN et al. The mouse tectorins. Modular matrix proteins of the inner ear homologous to components of the sperm-egg adhesion system. J. Biol. Chem. 272:8791-8801, 1997.

Legan PK, Lukashkina VA, Goodyear RJ et aL. A targeted deletion in alpha-tectorin reveals that the tectorial membrane is required for the gain and timing of cochlear feedback. Neuron 28:273285, 2000.

Legan PK, Lukashina VA, GoOdyear RJ ET aL. A deafness mutation isolates a second role for the tectorial membrane in hearing. Nat. Neurosci. 8:1035-1042, 2005.

McGuirt WT, Prasad SD, Griffith AJ et al. Mutations in COL11A2 cause non-syndromic hearing loss (DFNA13). Nat. Genet. 23:413-419, 1999.

Moore BCJ. Perceptual Consequences of Cochlear Damage. 1995.

Moser LM. [The Wurzburg auditory field, a test for prosthetic audiometry]. HNO 35:318-321, 1987.

Mustapha M, Chardenoux S, Nieder A et al. A sensorineural progressive autosomal recessive form of isolated deafness, DFNB13, maps to chromosome 7q34-q36. Eur. J. Hum. Genet. 6:245-250, 1998.

PAscoe DB. Clinical measurement of the auditory dynamic range and their relation to formulas for hearing aid gain. Proc. 13th Danavox symp.:1988. 
Pfister MHF, Thiele H, Van Camp G et al. A genotype-phenotype correlation with gender-effect for hearing impairment caused by TECTA mutations. Cell. Physiol. Biochem. 14:369-376, 2004.

Plantinga RF, de Brouwer AP, Huygen PlM et al. A Novel TeCTA Mutation in a Dutch DFNA8/12 family confirms genotypephenotype correlation. J. Assoc. Res. Otolaryngol. 7:173-181, 2006.

Plomp R, Mimpen AM. Improving the reliability of testing the speech reception threshold for sentences. Audiology 18:43-52, 1979.

Silman S, Gelfand SA. The relationship between magnitude of hearing loss and acoustic reflex threshold levels. J. Speech Hear. Disord. 46:312-316, 1981.

VAN CAMP G, Smith RJH. Hereditary Hearing Loss Homepage. http://webhost.ua.ac.be/hhh/ Accessed June 2006.

Verhoeven K, Van Camp G, Govaerts PJ et al. A gene for autosomal dominant nonsyndromic hearing loss (DFNA12) maps to chromosome 11q22-24. Am. J. Hum. Genet. 60:1168-1173, 1997.

Verhoeven K, Van Laer L, Kirschhofer K et al. Mutations in the human alpha-tectorin gene cause autosomal dominant nonsyndromic hearing impairment. Nat. Genet. 19:60-62, 1998. 